\title{
Nationwide assessment of the mental health of UK Doctoral Researchers
}

\author{
Cassie M. Hazell ${ }^{1}$, Jeremy E. Niven ${ }^{2}$, Laura Chapman ${ }^{3}$, Paul E. Roberts ${ }^{4}$, Sam Cartwright-Hatton ${ }^{3}$, \\ Sophie Valeix ${ }^{2} \&$ Clio Berry ${ }^{5}$
}

Doctoral Researchers (DRs) are an important part of the academic community and, after graduating, make substantial social and economic contributions. Despite this importance, DR wellbeing has long been of concern. Recent studies have concluded that DRs may be particularly vulnerable to mental health problems, yet direct comparisons of the prevalence of mental health problems between this population and control groups are lacking. Here, by comparing DRs with educated working controls, we show that DRs report significantly greater anxiety and depression, and that this difference is not explained by a higher rate of preexisting mental health problems. Moreover, most DRs perceive poor mental health as a 'normal' part of the PhD process. Thus, our findings suggest a hazardous impact of PhD study on mental health, with DRs being particularly at risk of developing common mental health problems. This provides an evidence-based mandate for universities and funders to reflect upon practices related to DR training and mental health. Our attention should now be directed towards understanding what factors may explain heightened anxiety and depression among DRs so as to inform preventative measures and interventions.

\footnotetext{
${ }^{1}$ School of Social Sciences, University of Westminster, 115 New Cavendish Street, London W1W 6UW, UK. ${ }^{2}$ School of Life Sciences, University of Sussex Falmer Brighton BN1 9QG, UK. ${ }^{3}$ School of Psychology, University of Sussex, Falmer Brighton BN1 9QJ, UK. ${ }^{4}$ Centre for Higher Education and Equity Research,

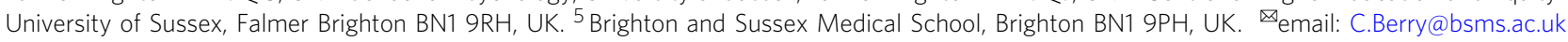




\section{Introduction}

niversity research makes a substantial contribution to the economy (University Alliance, 2014). A significant part of that contribution is driven by Doctoral Researchers (DRs), also known as $\mathrm{PhD}$ students, who consistently produce a reliable financial return on any investment in their studies, both to their institution and to industry more widely (Casey, 2009; EPSRC, 2010; Zolas et al., 2015). When asked during their PhD studies, the majority of DRs want to pursue a career in research post- $\mathrm{PhD}$ (Cornell, 2020). However, in reality, subsequent to receiving their $\mathrm{PhD}$, more than $70 \%$ leave academia completely (Hancock, 2020); instead pursuing careers in industry, government or non-profit organisations (Cornell, 2020). A key motivator for DRs leaving academia is to protect their mental health (Metcalfe et al., 2018). Therefore, the poor mental health of DRs and their subsequent exodus from academia will have implications for the volume and quality of academic research, as well as having broader social and economic impacts.

The issue of DR mental health was well evidenced in a recent international survey by Nature (Woolston, 2019). The survey found that $36 \%$ of current DRs reported seeking help for anxiety and/or depression (Woolston, 2019), with further editorials acknowledging the poor mental health of $\mathrm{PhD}$ students (Nature, 2019b, 2019a; Woolston, 2020). A recent meta-analysis found that DRs are moderately more stressed when compared to whole population normative data (Hazell et al., 2020). The available research all points in the same direction: DRs are stressed and experiencing poor mental health, and may be leaving the profession as a result of this (Hazell et al., 2020). However, there are significant limitations and gaps in the understanding of DR mental health (Hazell et al., 2020). In particular, we do not know whether DRs are at a higher risk of poor mental health than individuals who chose other career pathways because studies largely did not include comparison groups.

One recent study did attempt to address this gap in the field (Levecque et al., 2017); employing an online survey, this study revealed that DRs reported greater psychological distress than both undergraduate students and educated employees. However, this study, along with all others in the field (Hazell et al., 2020), did not assess symptoms indicative of Serious Mental Illness (SMI) diagnoses. Instead, this study (Levecque et al., 2017) used the General Health Questionnaire 12 (GHQ12) (Goldberg et al., 1997), which has been widely criticised for encouraging response biases, lack of reliability, and limited clinical validity (Hankins, 2008a, 2008b; Ye, 2009). Finally, to our knowledge, no studies have addressed the issue of causality. That is, whether those with existing poor mental health might be more attracted to pursuing $\mathrm{PhD}$ studies or whether studying for the $\mathrm{PhD}$ itself is the cause of mental health difficulties.

To address these limitations, we conducted a UK-based mixedmethods online survey with DRs, and a control group comprised of similarly aged, educated working professionals (WP). The central aim of our study was to determine how the prevalence of mental health problems differs between DRs and WPs. Secondary to this, we aimed to assess DRs' perceptions of the commonality of mental health problems. Our survey employed outcomes measures with well-established clinical cut-offs and included measures that capture SMI symptoms i.e. mania and suicidality. We also controlled for pre-existing mental health problems, which allowed us to test whether our data support PhD study as causative of mental health problems.

\section{Methods}

Participants. We recruited DRs and a matched group of educated WPs. To be eligible for inclusion in the DR group, participants had to be currently studying for their PhD at a UK University. The eligibility criteria for the WP group were developed to ensure similarity between the two groups, other than $\mathrm{PhD}$ status. Eligibility criteria, therefore, reflected the minimum entry requirements for PhD programmes in the UK. WPs had to be aged 21 years or over and possess a university undergraduate degree of 2.1 or above. Moreover, WPs had to be working in the UK at least 3 days a week ( 0.6 full time equivalent (FTE)) as this matched the minimum FTE for a part-time PhD.

Recruitment. To recruit DRs, we emailed every Doctoral School in the UK $(N=162)$ asking them to share details of the study with their students. To recruit WPs, we emailed the public relations departments of the top 100 graduate employers and the top 500 UK businesses. None of the graduate employers confirmed that they had disseminated the survey details. Additionally, we promoted the study via the project social media outlets, Prolific Academic and paid advertisements on Facebook. As part of the debrief information, we asked participants to share details of the survey with their personal and professional networks.

Design. The study is an online, cross-sectional survey. We used a between-group design, comparing responses from DRs to WPs. The survey was administered using Qualtrics survey software.

Measures. We collected data using a battery of measures of mental health problems and psychological and social functioning. In the present paper, we report comparative data on prevalence and symptomatology for DRs and WPs.

Demographics. Participants were asked to self-identify as either a DR or a WP and then complete basic demographic questionnaires. DRs were asked for information about their $\mathrm{PhD}$, including funding status and whether they were engaging in fieldwork. WPs were asked to classify the type of job they have and how likely they were to complete a $\mathrm{PhD}$ in the future.

As part of the demographic questionnaire, we asked participants about their mental health, including whether they have a mental health diagnosis, and whether they have experienced a mental health crisis. With the aim of assessing premorbid mental health, we adapted interview questions from the Composite International Diagnostic Interview (CIDI) (Kessler and Üstün, 2004), in which lifetime prevalence participants are asked to reflect on key milestones across the lifespan and whether they were experiencing mental health problems at that time. Using milestones to determine the onset of mental health problems is associated with increased accuracy of retrospective data (Kessler et al., 2005). We provided participants with a series of milestones related to their studies and work history and asked them to indicate whether they were experiencing mental health problems at that time or not, for example while at secondary school, before they started an undergraduate degree, while completing an undergraduate degree and so on.

Perceived mental health problem commonality and impact. We asked DR participants only to respond to several statements assessing DRs' perceptions of the commonality and impact of mental health problems, for example, whether they thought it was the norm to experience mental health difficulties during a $\mathrm{PhD}$, and whether they had considered or had actually taken time away from their studies in relation to mental health. These statements had the following response options: true, false, or not sure.

Patient Health Questionnaire 9 (PHQ-9). The PHQ-9 is widely used in UK primary healthcare as a depression screening tool 
(Spitzer et al., 1999). The PHQ-9 has 9 items which are scored by participants using a 4-point Likert scale from 'not at all' (0) to 'nearly every day' (4). A higher score indicates greater depression symptoms. The scale has strong internal consistency (Cronbach's $\alpha=0.89$ ) and well-established cut-off criteria delineating clinical from sub-clinical levels of depression (Kroenke et al., 2001). In the present sample, we also found the PHQ- 9 to have good scale reliability (Cronbach's $\alpha=0.89$ ).

Generalised Anxiety Disorder 7 (GAD-7). Akin to the PHQ-9, the GAD-7 (Spitzer et al., 2006) is used in UK primary healthcare settings as a screening tool for anxiety/GAD. The GAD-7 has 7 items, scored by participants on a 4 -point Likert scale, ranging from 'not at all' (0) to 'nearly every day' (4). A higher score indicates greater anxiety symptoms. The scale has strong internal consistency (Cronbach's $\alpha=0.92$ ) with well-established cut-off scores distinguishing those with clinical and sub-clinical levels of anxiety (Spitzer et al., 2006). We found similar levels of scale reliability in the current sample (Cronbach's $\alpha=0.90$ ).

Altman Self-Reported Mania Scale (ASRMS). The 5-item ASRMS measures symptoms of mania (Altman et al., 1997). Each item has a 5-point Likert scale with unique anchor points. A higher score indicates greater mania. The scale has acceptable levels of internal consistency (Cronbach's $\alpha=0.65$ ) (Altman et al., 1997), which was replicated in the current sample (Cronbach's $\alpha=0.66$ ). The scale reliability could not be improved by removing any of the items.

Suicidal Behaviours Questionnaire-Revised (SBQ-R). The SBQ-R (Osman et al., 2001a) comprises four items; each captures a different facet of suicidality and all combine to provide an assessment of overall suicide risk. Each of the items has its own Likert Scale and scoring system. A higher score indicates greater suicide risk. The SBQ-R has good internal consistency (all Cronbach $\alpha s>0.70$ ), with a score of 7 or above amongst a general population sample indicating a high risk of suicide (Osman et al., 2001a). The scale reliability found here exceeds that reported by Osman et al. (2001a, 2001b) (Cronbach's $\alpha=0.81$ ).

Procedure. Participants were able to access the online survey via a weblink provided. After being presented with the participant information screen, participants were asked to provide consent by completing a 'tick box' form. Participants were next asked to complete an eligibility screening questionnaire. Eligible participants were then invited to complete the questionnaire battery. Because of the sensitive nature of the measures, all of the questions were optional. At the end of the survey, participants were presented with debriefing information and invited to enter a prize draw for one of two iPad minis.

Ethics. This study received ethical approval from the University of Sussex Sciences and Technology Cross-Schools Research Ethics Committee (C-REC) on the 19 December 2017 (Reference: ER/ $\mathrm{CH} 283 / 9)$.

Analysis plan. Data analyses were conducted using SPSS version 25. We first calculated the descriptive statistics for all of the demographic items and clinical measures. Means and standard deviations are provided for continuous variables, and frequencies and percentages are given for categorical variables. Missing data were examined by performing a forced entry logistic regression with complete (complete questionnaire battery) versus missing data (demographic data present only) as the outcome variable, and key demographics entered as predictors. Specifically, we included age, and dummy codes for gender (females versus all other genders, males versus all other genders, and other gender versus male or female), ethnicity (White British versus all other ethnicities, and White versus all other ethnicities), disability (versus no disability), and profession (DRs versus WPs) as predictors. None of the sample characteristics significantly predicted whether data were missing or complete (i.e. age, female versus non-females, males versus non-male, other gender versus nonother, White British versus non-White British, White versus nonWhite, disabled versus not disabled, and group; all $p s>0.05$ ).

Our research questions were tested first by calculating the frequencies and percentages of DRs that endorsed specific statements related to mental health. Second, we conducted an independent samples $t$-test comparing the reported age of mental health problem onset between DRs and WPs. This analysis was supplemented with chi-square tests to compare the milestone (life phase) at which symptoms reportedly emerged between DRs and WPs. Where any significant interactions were identified, these were further explored using the distribution of counts.

Finally, we performed a one-way independent-groups MANCOVA with a Bonferroni correction. Our grouping variable compared DRs and WPs. We entered four dependent variables into the model: depression (PHQ-9 total), anxiety (GAD-7 total), mania (ASMRS total), and suicidality (SBQ-R total). To control for confounding variables, we entered age, gender (dummy coded as female versus male and other; male versus female and other; and other versus female and male), disability status (reporting a disability versus not), ethnicity (White British versus all other ethnicities; and White versus non-White), and mental health history (reported a history of mental health problems versus no history) as covariates.

\section{Results}

Perceived mental health problem commonality and impact. In total, 4608 participants were included in the analysis of whom 3352 were DRs and 1256 WPs (see Fig. 1 and Table 1). Based on figures from the Higher Education Statistics Agency (HESA, 2019), our sample represented $\sim 3.29 \%$ of the total UK DR population. The majority of DRs agreed that developing a mental health problem during a $\mathrm{PhD}$ is 'the norm' and that most of their peers had experienced mental health problems (Table 2). More than a third of DRs (35.8\%) had considered ending and/or taking a break from their studies due to poor mental health. Just over

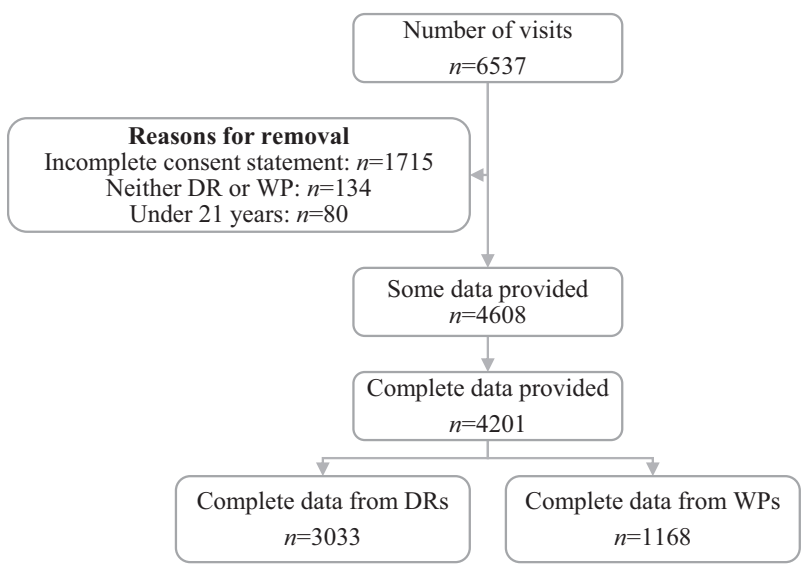

Fig. 1 CONSORT diagram of sample sizes and reasons that data was removed. Note: Complete data here is classed as any participant where they provided some level of demographic data and complete data for at least one of the clinical outcome measures. WP working professionals, DR doctoral researchers. 
Table 1 Sample characteristics for the total sample and separated by DRs versus WPs.

\begin{tabular}{|c|c|c|c|c|c|c|}
\hline & \multicolumn{2}{|l|}{ All } & \multicolumn{2}{|l|}{ DRs } & \multicolumn{2}{|l|}{ WPs } \\
\hline \multicolumn{7}{|l|}{ General } \\
\hline Gender & 4607 & & 3352 & & 1255 & \\
\hline Male & & 1563(33.9) & & 1102(32.9) & & 461(36.7) \\
\hline Female & & $2987(64.8)$ & & $2205(65.8)$ & & $782(62.3)$ \\
\hline Ethnicity & 4606 & & 3351 & & 1255 & \\
\hline White British & & $2457(53.3)$ & & $1749(52.2)$ & & 708(56.4) \\
\hline White Other & & $1356(29.4)$ & & $938(28.0)$ & & $418(33.3)$ \\
\hline Chinese/Chinese British & & $82(1.8)$ & & $68(2.0)$ & & $14(1.1)$ \\
\hline Black/African/Caribbean/Black British & & $100(2.2)$ & & $76(2.3)$ & & 24(1.9) \\
\hline Asian/Asian British & & $258(5.6)$ & & $216(6.4)$ & & 42(3.3) \\
\hline No & & 1621(35.3) & & $1229(36.8)$ & & 392(31.3) \\
\hline English first language & 4597 & & 3344 & & 1253 & \\
\hline Yes & & $3141(68.3)$ & & $2285(68.3)$ & & $856(68.3)$ \\
\hline No & & $1456(31.7)$ & & $1059(31.7)$ & & $397(31.7)$ \\
\hline Dependents & 4594 & & 3341 & & 1253 & \\
\hline Yes & & $1040(22.6)$ & & $643(19.2)$ & & $397(31.7)$ \\
\hline No & & $3554(77.1)$ & & 2698(80.8) & & $856(68.3)$ \\
\hline Completed a Postgraduate Taught (PGT) qualification & 4590 & & 3339 & & 1251 & \\
\hline Yes & & $3255(70.9)$ & & 2734(81.9) & & $521(41.6)$ \\
\hline No & & 1335(29.1) & & $605(18.1)$ & & $730(58.4)$ \\
\hline Location & 3967 & & 2783 & & 1184 & \\
\hline South East of England & & $588(14.8)$ & & 296(10.6) & & 292(24.7) \\
\hline Northern Ireland & & 138(3.5) & & $114(4.1)$ & & $24(2.0)$ \\
\hline Wales & & 101(2.6) & & $74(2.7)$ & & $27(2.3)$ \\
\hline Disability & 4328 & & 3120 & & 1208 & \\
\hline Yes & & $682(15.8)$ & & $506(16.2)$ & & $176(14.6)$ \\
\hline No & & $3517(81.3)$ & & $2517(80.7)$ & & $1000(82.8)$ \\
\hline $\begin{array}{l}\text { Prefer not to say } \\
\text { Disability type }\end{array}$ & & $129(3.0)$ & & $97(3.1)$ & & $32(2.6)$ \\
\hline Blind or sight loss & & 19 & & 9 & & 10 \\
\hline Deaf or hearing loss & & 19 & & 12 & & 7 \\
\hline Mobility issues & & 59 & & 41 & & 18 \\
\hline Manual dexterity & & 22 & & 16 & & 6 \\
\hline Learning disability & & 129 & & 107 & & 22 \\
\hline Speech impairment & & 6 & & 2 & & 4 \\
\hline Cognitive disability & & 79 & & 62 & & 17 \\
\hline Other & & 176 & & 113 & & 63 \\
\hline Prefer not to say & & 11 & & 9 & & 2 \\
\hline $\begin{array}{l}\text { Area of study (PhD studies or undergraduate studies } \\
\text { for WPs) }\end{array}$ & 4268 & & 3089 & & 1179 & \\
\hline Arts & & $388(9.1)$ & & $255(8.3)$ & & 133(11.3) \\
\hline Biological and medical sciences & & 701(16.4) & & 614(19.9) & & $87(7.4)$ \\
\hline Business and finance & & $274(6.4)$ & & $135(4.4)$ & & 139(11.8) \\
\hline Chemical sciences & & $104(2.4)$ & & $93(3.0)$ & & $11(0.9)$ \\
\hline Earth sciences & & $145(3.4)$ & & 118(3.8) & & $27(2.3)$ \\
\hline Engineering & & 293(6.9) & & $160(5.2)$ & & 133(11.3) \\
\hline Humanities & & $566(13.3)$ & & $420(13.6)$ & & $146(12.4)$ \\
\hline
\end{tabular}


Table 1 (continued)

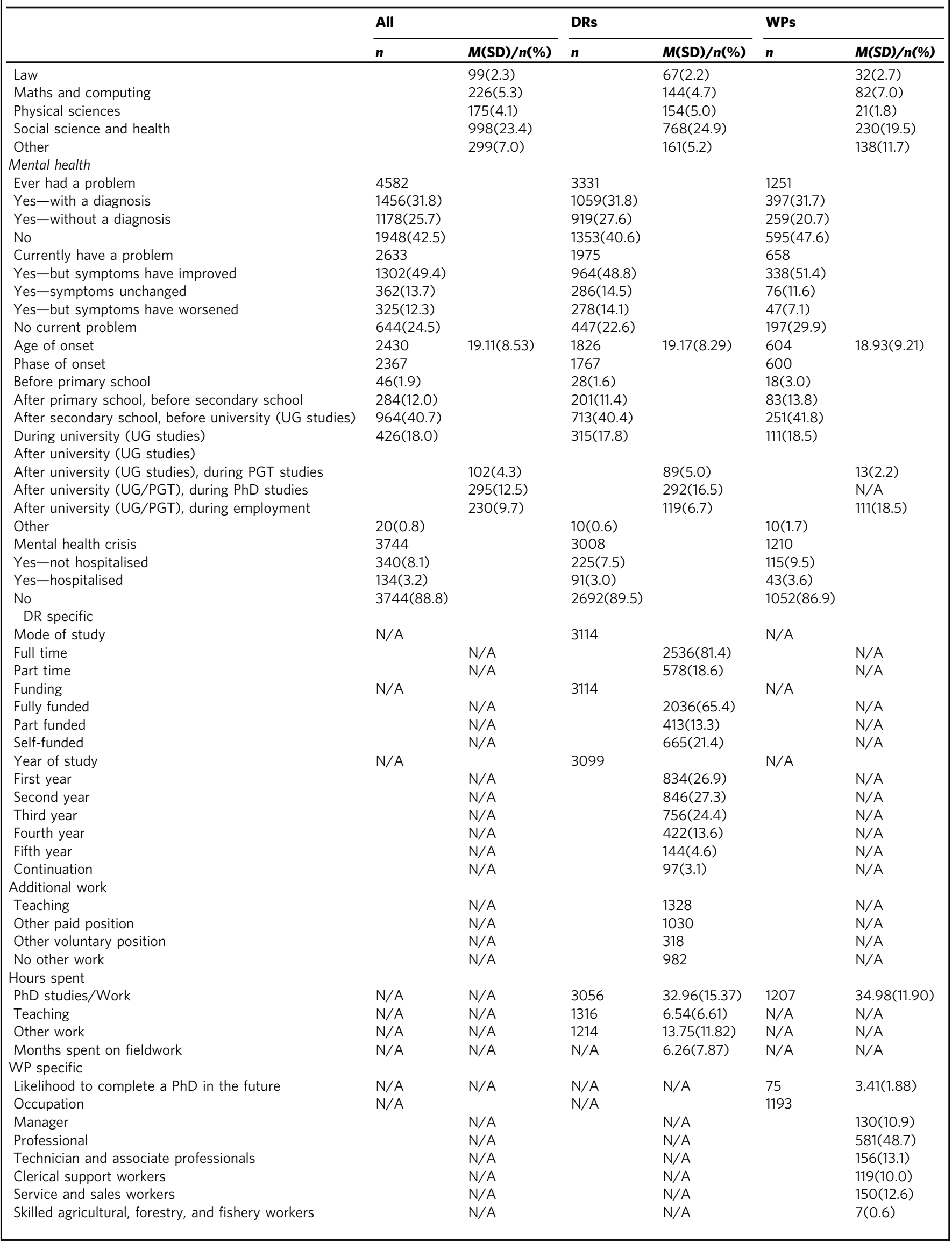


Table 1 (continued)

\begin{tabular}{|c|c|c|c|c|c|c|}
\hline & \multicolumn{2}{|c|}{ All } & \multicolumn{2}{|c|}{ DRs } & \multicolumn{2}{|c|}{ WPs } \\
\hline Craft and related trade workers & & N/A & & N/A & & 23(1.9) \\
\hline Elementary occupations & & $\mathrm{N} / \mathrm{A}$ & & $\mathrm{N} / \mathrm{A}$ & & $15(1.3)$ \\
\hline Armed forces occupations & & N/A & & N/A & & $7(0.6)$ \\
\hline
\end{tabular}

$D R$ doctoral researchers, WP working professionals, N/A not applicable-the question is not applicable to that participant group, $n$ number of participants, $M$ mean, SD standard deviation.

Table 2 The percentage of DRs who endorsed statements related to experiencing mental health problems while studying for a PhD.

\begin{tabular}{llccc} 
& Total $\boldsymbol{n}$ & True $\boldsymbol{n ( \% )}$ & False $\boldsymbol{n ( \% )}$ & Not sure $\boldsymbol{n ( \% )}$ \\
\hline I have considered ending my PhD studies because of my mental health & 3060 & $1097(35.8)$ & $1654(54.1)$ & $309(10.1)$ \\
I have had to take a break from my PhD studies because of mental health & 3059 & $455(14.6)$ & $2461(80.5)$ & $143(4.7)$ \\
I have considered taking a break from my PhD studies because of my mental health & 3057 & $1283(42.0)$ & $1626(53.2)$ & $148(4.8)$ \\
I think developing a mental health problem during your PhD is the norm & 3058 & $1293(42.3)$ & $988(32.3)$ & $777(25.4)$ \\
Most of my peers doing PhDs have experienced mental health problems & 3056 & $1240(40.6)$ & $610(20.0)$ & $1206(39.5)$ \\
\hline
\end{tabular}

Table 3 Descriptive statistics for all clinical outcome measures for all participants and separated by DRs and WPs.

\begin{tabular}{|c|c|c|c|c|c|c|}
\hline & \multicolumn{2}{|l|}{ All } & \multicolumn{2}{|l|}{ DRs } & \multicolumn{2}{|l|}{ WPs } \\
\hline \multicolumn{7}{|l|}{ Scores } \\
\hline GAD-7 Toal & 4201 & $8.20(5.46)$ & 3033 & $8.73(5.56)$ & 1168 & $6.82(4.92)$ \\
\hline ASMRS Total & 4146 & $3.19(2.54)$ & 2985 & $3.05(2.44)$ & 1161 & $3.54(2.76)$ \\
\hline SBQ-R Total & 3872 & $5.95(3.40)$ & 2770 & $5.60(3.40)$ & 1102 & $5.83(3.38)$ \\
\hline None & & $1313(31.4)$ & & $875(29.1)$ & & $438(37.5)$ \\
\hline Mild & & $1237(29.6)$ & & $882(29.3)$ & & $355(30.4)$ \\
\hline Moderate & & $822(19.7)$ & & $628(20.9)$ & & $194(16.6)$ \\
\hline Moderately severe & & $494(11.8)$ & & $375(12.5)$ & & $119(10.2)$ \\
\hline Severe & & $312(7.5)$ & & $251(8.3)$ & & $61(5.2)$ \\
\hline GAD-7 & 4201 & & 3033 & & 1168 & \\
\hline No mania & & $3420(82.5)$ & & $2526(84.6)$ & & $894(77.0)$ \\
\hline High probability of mania & & 726(17.5) & & $459(15.4)$ & & $267(23.0)$ \\
\hline SBQ-R & 3872 & & 2770 & & 1102 & \\
\hline No risk & & $2533(65.4)$ & & $1797(64.9)$ & & $736(66.8)$ \\
\hline At risk & & $1339(34.6)$ & & 973(35.1) & & $366(33.2)$ \\
\hline
\end{tabular}

$14 \%$ of DRs had formally intermitted due to mental health problems.

Lifetime prevalence. DRs reported a greater lifetime prevalence of mental health difficulties than WPs $\left(\chi^{2}(1)=18.39, p<0.001\right.$; $\left.\varphi_{c}=0.06\right)$, but this difference was no longer present when considering only a history of professionally diagnosed mental health problems $\left(\chi^{2}(1)=0.00, p=0.99 ; \varphi_{c}<0.01\right)$.
Timing of symptom onset. There was no difference between DRs and WPs in terms of the age at which mental health problems began $(t(946.51)=0.58, p=0.56)$ (Table 3$)$. However, there was a small significant difference between DRs and WPs in terms of the life phase at which mental health problems first emerged $\left(\chi^{2}(5)=26.09, p<0.001 ; \varphi_{c}<0.10\right)$. Compared to WPs, DRs generally had a later onset of mental health problems (i.e. starting during or after undergraduate studies). This association remained when the analysis was replicated with participants who did 
$\left(\chi^{2}(5)=14.16, p=0.02 ; \varphi_{c}<0.10\right)$ or did not report having been given a formal mental health problem diagnosis $\left(\chi^{2}(5)=12.82\right.$, $\left.p=0.03 ; \varphi_{c}<0.10\right)$.

\section{Prevalence of mental health problems}

Clinical cut-offs. The prevalence of probable depressive disorders, as assessed using the PHQ9 (Spitzer et al., 1999) $\left(\chi^{2}(4)=41.74\right.$, $\left.p<0.001 ; \varphi_{c}=0.10\right)$, and prevalence of probable Generalised Anxiety Disorder (GAD) $\left(\chi^{2}(3)=98.47, p<0.001 ; \varphi_{c}=0.15\right)$, as assessed using the GAD7 (Spitzer et al., 2006), was significantly greater amongst DRs than WPs. Moderate to severe presentations of depression and anxiety were more common amongst DRs, whereas having no or mild symptoms was more typical amongst WPs.

By contrast, the probable risk of mania, as measured by the ASRMS (Altman et al. 1997), was greater amongst WPs than DRs $\left(\chi^{2}(1)=33.61, p<0.001 ; \varphi_{c}=0.09\right)$. That is, a high probability of mania was more prevalent amongst WPs whereas being at no risk of mania was more common amongst DRs. There was no difference between WPs and DRs in terms of suicide risk as measured using the Suicidal Behaviours Questionnaire-Revised (SBQ-R) (Osman et al., 2001a) $\left(\chi^{2}(1)=1.28, p=0.26 ; \varphi_{c}=0.02\right)$.

Symptom severity. When turning to mental health symptoms (as opposed to probable diagnoses), overall symptomology was significantly greater for DRs compared to WPs $(\Lambda=0.98$, $F(4,3737)=22.60, \quad p<0.001)$. Specifically, the severity of depression $(F(1,3740)=14.97, p<0.001 ; \mathrm{DR}: \mathrm{EMM}=8.77$, $\mathrm{SE}=0.11 ; \mathrm{WP}: \mathrm{EMM}=7.96, \mathrm{SE}=0.18)$ and anxiety symptoms $(F(1,3740)=57.58, p<0.001$; DRs: $\mathrm{EMM}=8.47, \mathrm{SE}=0.10$; WP: $\mathrm{EMM}=7.08, \mathrm{SE}=0.16)$ were significantly greater for DRs compared to WPs. Conversely, symptoms of mania were greater in WPs compared to DRs $(F(1,3740)=27.97, p<0.001$; WPs: $\mathrm{EMM}=3.53, \mathrm{SE}=0.08$; $\mathrm{DRs}: \mathrm{EMM}=3.06, \mathrm{SE}=0.05$ ), and there was no difference between the groups in terms of suicidality $(F(1,3740)=0.39, p=0.53$; DRs: $\mathrm{EMM}=5.92, \mathrm{SE}=0.06$; WPs: $\mathrm{EMM}=5.98, \mathrm{SE}=0.09$ ).

Item-level analysis of suicidality. The SBQ-R (Osman et al., 2001a) measures overall suicide risk with individual items assessing various facets of suicidality (i.e. lifetime suicidal thoughts, recent suicidal thoughts, future suicide intent, and suicide behaviours). WPs (EMM $=1.30, \mathrm{SE}=0.02)$ were more likely to report having attempted suicide than $\mathrm{DRs}(\mathrm{EMM}=1.26$, $\mathrm{SE}=0.01)(F(2,3748)=5.72, p=0.02)$. There was no difference between DRs and WPs in terms of suicidal thoughts (lifetime or recent) or future suicide risk (lifetime suicidal thoughts: DRs: $\mathrm{EMM}=1.85, \mathrm{SE}=0.02$; WPs: $\mathrm{EMM}=1.90, \mathrm{SE}=0.03 ; p=0.16$; recent suicidal thoughts: $\mathrm{DRs}: E M M=1.82, \mathrm{SE}=0.02$; WPs: $\mathrm{EMM}=1.81, \mathrm{SE}=0.04$; future suicide risk: $\mathrm{DRs}: \mathrm{EMM}=0.99$, $\mathrm{SE}=0.02$; WPs: $\mathrm{EMM}=0.98, \mathrm{SE}=0.04 ; p=0.84$ ).

\section{Discussion}

We aimed to ascertain whether, compared to an educated working control group, DRs were at significantly greater risk for both common (depression and anxiety) and serious (mania and suicidality) mental health problems and whether any greater risk was explained by pre-existing differences in mental health problem proneness. We also aimed to understand DRs' beliefs regarding the commonality and occupational impact of problems with their mental health. DRs reported significantly higher levels of depression and anxiety, and significantly lower levels of mania, than WPs. There was no main difference between DRs and WPs in reported suicidality, but our item-level exploratory analysis of facets of suicidality revealed that WPs reported greater risk for engaging in suicide behaviours. DRs widely endorsed beliefs about the normalcy of mental health problems in their peer group. Moreover, there appeared to be an impact of this poor mental health on considered and actual intermissions from $\mathrm{PhD}$ study.

When viewed alongside those of Levecque et al. (2017), our results suggest that mental health problems are more prevalent amongst UK-based DRs than Belgian DRs. More than half of DRs (51.1\%) in Belgium met the criteria for psychological distress (Levecque et al., 2017); where we found that $70.9 \%$ and $74.2 \%$ of DRs reported clinically relevant (i.e. mild to severe symptoms) of depression and anxiety symptoms, respectively. The difference between these findings could reflect differences in the outcome measures employed; Levecque et al. (2017) used the General Health Questionnaire (GHQ) (Goldberg, 1972), whereas we used the PHQ-9 (Spitzer et al., 1999) and GAD-7 (Spitzer et al., 2006). Alternatively, this could represent genuine differences in the prevalence of mental health problems across countries, over time, or with respect to the conditions DRs experience during their $\mathrm{PhD}$.

The high rates of depression and anxiety symptoms within our UK DR sample are concerning. In addition to the clear distress reported here, depression and anxiety symptoms are likely to undermine the chances of successfully completing a $\mathrm{PhD}$ by impairing academic performance via undermining neurocognitive functioning (Castaneda et al., 2008), for example through impairing executive functioning, attention, and working memory (Shackman et al., 2006; Rock et al., 2014; Shields et al., 2016). Supporting the mental health of DRs is important to not only improve the wellbeing of these students, but also to enable them to successfully complete their studies and ensure important contributions to the research (Levecque et al., 2017) and knowledge economies (Kehm, 2006).

The overall scores from our measure of suicidality (using the SBQ-R (Osman et al., 2001a)) yielded no significant differences between DRs and WPs. However, closer inspection of the scores reveals a worrying picture, whereby both groups reported alarmingly high levels of suicide risk. A concerning $35 \%$ of DRs (and WPs) met criteria for 'suicide risk', using measure thresholds derived for the general population (Osman et al., 2001b). There have been two studies that have used the SBQ- $R$ to assess suicidality in the general population (Castillejos et al., 2020). The prevalence of suicide risk in both of our samples far exceeds the prevalence observed in the general population studies of 4.6\% (Polatöz et al., 2011; Blüml et al., 2013) at its highest. This high suicide risk may be a consequence of the high prevalence of depression in our sample, which could therefore be reduced by ameliorating depressive symptoms, though this relationship requires testing. Currently, national suicide data for all UK students is collected by the Higher Education Statistics Agency (HESA) and the Office for National Statistics (ONS). To help identify which students are at greatest risk of dying by suicide, we recommend these data differentiate and disaggregate by phase of study so that suicide rates are monitored amongst DRs specifically.

There was no difference in terms of the lifetime prevalence of a diagnosed mental health problem or the age of symptom onset between DRs and WPs. However, WPs generally reported mental health problems starting in an earlier life phase than did DRs. These data combined suggests that those with long-term mental health problems are not disproportionately drawn to study for a $\mathrm{PhD}$ and that the high prevalence of mental health problems amongst DRs does not simply represent pre-existing conditions. 
Limitations. Despite efforts to recruit both those with and without mental health difficulties, our results may be skewed by a selection bias. That is, the survey may have disproportionately attracted respondents within both the DR and the educated working control group who have mental health problems (with or without diagnosis) compared to those who do not. Mental health prevalence statistics for the UK general population suggest $25 \%$ (1 in 4) (Time to Change, 2015) of people experience mental health problems; whereas, in the present sample, prevalence was more than double this (59.4\% for DRs and $52.4 \%$ for WPs). Although this may limit the representativeness of our findings, it does in fact strengthen our conclusions. That is, even compared to a WP sample who have particularly high rate of mental health problems and which had an earlier onset, DRs still report significantly greater levels of depression and anxiety. Similarly, our findings may have limited generalisability due to the disproportionate number of White British and female participants who participated in the survey and is not necessarily being applicable beyond the UK.

Our survey used brief, self-report measures of mental health problems, which may be more likely to produce less reliable and valid results than a diagnostic interview. However, there is some suggestion that using measures such as these, particularly in an online setting, reduces social desirability (Henderson et al., 2012; Zhang et al., 2017) and therefore, our findings may actually better reflect prevalence than if we had interviewed participants face-to-face.

We must also acknowledge the limitations of our exploratory post-hoc analysis of the SBQ-R (Osman et al., 2001a) data. Suicidality can broadly be conceptualised as thoughts (e.g. ideation) and action (e.g. planning and attempts) (Klonsky et al., 2016), and after examining full-scale scores we decided to carry out the additional item-level analysis. As this item level analysis was not planned a priori, the results should be interpreted with caution.

Research implications. In addition to measuring common mental health symptoms (depression and anxiety), we included measures related to more SMI conditions (mania and suicide). Yet there remains a need to explore other SMI symptoms and unusual experiences. Moreover, DRs are a heterogenous group, for example in relation to funding, area of study, and home/ international status, and we did not explore associations between such characteristics and mental health problems. Furthermore, while our findings suggest that DRs generally have poorer mental health, we have not drawn any conclusions as to putative mechanisms that explain this difference. We have however begun to test predictive associations of DR mental health symptoms in a further study (Berry et al., 2021).

\section{Data availability}

The data are available upon reasonable request to the corresponding author.

Received: 4 December 2020; Accepted: 13 September 2021; Published online: 09 December 2021

\section{References}

Altman EG, Hedeker D, Peterson JL, Davis JM (1997) The Altman self-rating Mania scale. Biol Psychiatry 42:948-955. https://doi.org/10.1016/S00063223(96)00548-3

Berry C, Niven J, Hazell C (2021) Personal, social and relational predictors of UK postgraduate researcher mental health problems. BJPsych Open, 7:E205. https://doi.org/10.1192/bjo.2021.1041
Blüml V, Kapusta ND, Doering S et al. (2013) Personality factors and suicide risk in a representative sample of the German general population. PLoS ONE 8:1-7. https://doi.org/10.1371/journal.pone.0076646

Casey BH (2009) The economic contribution of PhDs. J High Educ Policy Manag 31:219-227. https://doi.org/10.1080/13600800902974294

Castaneda AE, Tuulio-Henriksson A, Marttunen M et al. (2008) A review on cognitive impairments in depressive and anxiety disorders with a focus on young adults. J Affect Disord 106:1-27. https://doi.org/10.1016/j.jad.2007.06.006

Castillejos MC, Huertas P, Martín P, Moreno Küstner B (2020) Prevalence of suicidality in the european general population: a systematic review and meta-analysis. Arch Suicide Res 0:1-19. https://doi.org/10.1080/13811118.2020.1765928

Cornell B (2020) PhD students and their careers. London, UK

Engineering \& Physical Science Research Council (EPSRC) (2010) The economic impact of PhDs. Edinburgh

Goldberg DP (1972) The detection of psychiatric illness by questionnaire. Maudsley Monogr 21: Oxford University Press

Goldberg DP, Gater R, Sartorius N et al. (1997) The validity of two versions of the GHQ in the WHO study of mental illness in general health care. Psychol Med 27:191-197. https://doi.org/10.1017/S0033291796004242

Hancock S (2020) The employment of $\mathrm{PhD}$ graduates in the UK: what do we know? Higher Education Policy Institute. https://www.hepi.ac.uk/2020/02/ 17/the-employment-of-phd-graduates-in-the-uk-what-do-we-know/. Accessed 22 Oct 2020

Hankins M (2008a) Clinical Practice and Epidemiology Executive function abnormalities in pathological gamblers. Clin Pract Epidemiol Ment Health 6:1-6. https://doi.org/10.1186/1745-0179-4-Received

Hankins M (2008b) The reliability of the twelve-item general health questionnaire (GHQ-12) under realistic assumptions. BMC Public Health 8:1-7. https:// doi.org/10.1186/1471-2458-8-355

Hazell CM, Chapman L, Valeix SF et al. (2020) Understanding the mental health of doctoral researchers: a mixed methods systematic review with meta-analysis and meta-synthesis. Syst Rev 9:1-30

Henderson C, Evans-Lacko S, Flach C, Thornicroft G (2012) Responses to mental health stigma questions: the importance of social desirability and data collection method. Can J Psychiatry 57:152-160. https://doi.org/10.1177/ 070674371205700304

HESA (2019) Who's studying in HE? Higher Education Statistics Agency. https:// www.hesa.ac.uk/data-and-analysis/students/whos-in-he. Accessed 3 Nov 2020

Kehm BM (2006) Doctoral education in Europe and North America: a comparative analysis. In: Teichler U (ed) The formative years of scholars. Wenner-Gren International Series. Portland Press, London, UK, pp. 67-78

Kessler RC, Berglund P, Demler O et al. (2005) Lifetime prevalence and age-ofonset distributions of DSM-IV disorders in the National Comorbidity Survey Replication. Arch Gen Psychiatry 62:593-602. https://doi.org/10.1001/ archpsyc.62.6.593

Kessler RC, Üstün BB (2004) The World Mental Health (WMH) Survey Initiative version of the World Health Organization (WHO) Composite International Diagnostic Interview (CIDI). Int. J. Methods Psychiatr. Res. 13:93-117

Klonsky ED, May AM, Saffer BY (2016) Suicide, suicide attempts, and suicidal ideation. Annu Rev Clin Psychol 12:307-330. https://doi.org/10.1146/ annurev-clinpsy-021815-093204

Kroenke K, Spitzer RL, Williams JBW (2001) The PHQ-9: validity of a brief depression severity measure. J Gen Intern Med 16:606-613. https://doi.org/ 10.1046/j.1525-1497.2001.016009606.x

Levecque K, Anseel F, De Beuckelaer A et al. (2017) Work organization and mental health problems in $\mathrm{PhD}$ students. Res Policy 46:868-879. https://doi.org/ 10.1016/j.respol.2017.02.008

Metcalfe J, Wilson S, Levecque K (2018) Exploring wellbeing and mental health and associated support services for postgraduate researchers. Cambridge

Nature (2019a) The mental health of PhD researchers demands urgent attention. Nature 575:257-258. https://doi.org/10.1038/d41586-019-03489-1

Nature (2019b) Academia's mental-health woes. Nature 569:307

Osman A, Bagge CL, Gutierrez PM et al. (2001a) The Suicidal Behaviors Questionnaire-Revised (SBQ-R): validation with clinical and nonclinical samples. Assessment 8:443-454. https://doi.org/10.1177/107319110100800409

Osman A, Bagge CL, Gutierrez PM et al. (2001b) The Suicidal Behaviors Questionnaire-Revised (SBQ-R): validation with clinical and nonclinical samples. Assessment 8:443-454

Polatöz Ö, Kuğu N, Doğan O, Akyüz G (2011) The prevalence of suicidal behaviour and its correlation with certain sociodemographic variables in Sivas province. Dusunen Adam J Psychiatry Neurol Sci 24:13-23. https://doi.org/ 10.5350/dajpn2011240102

Rock PL, Roiser JP, Riedel WJ, Blackwell AD (2014) Cognitive impairment in depression: a systematic review and meta-analysis. Psychol Med 44:2029-2040. https://doi.org/10.1017/S0033291713002535

Shackman AJ, Sarinopoulos I, Maxwell JS et al. (2006) Anxiety selectively disrupts visuospatial working memory. Emotion 6:40-61. https://doi.org/10.1037/ 1528-3542.6.1.40 
Shields GS, Moons WG, Tewell CA, Yonelineas AP (2016) Anxiety not anger impairs executive functionioning. Emotion 16:792-797. https://doi.org/ 10.1016/j.antiviral.2015.06.014.Chronic

Spitzer RL, Kroenke K, Williams JBW et al. (2006) A brief measure for assessing generalized anxiety disorder. Arch Intern Med 166:1092. https://doi.org/ 10.1001/archinte.166.10.1092

Spitzer RL, Kroenke K, Williams JBW, Group PHQPCS (1999) Validation and utility of a self-report version of PRIME-MD: the PHQ Primary Care Study. JAMA 282:1737. https://doi.org/10.1001/jama.282.18.1737

Time to Change (2015) Attitudes to mental illness 2014 research report. Time to Change, London

University Alliance (2014) The contribution of university research to economic growth. University Alliance, London, UK

Woolston C (2019) $\mathrm{PhD}$ poll reveals fear and joy, contentment and anguish. Nature 575:403-406. https://doi.org/10.1038/d41586-019-03459-7

Woolston C (2020) Pandemic and panic for US graduate students. Nature 585:147-148. https://doi.org/10.1038/d41586-020-02439-6

Ye S (2009) Factor structure of the General Health Questionnaire (GHQ-12): The role of wording effects. Pers Individ Dif 46:197-201. https://doi.org/10.1016/ j.paid.2008.09.027

Zhang XC, Kuchinke L, Woud ML et al. (2017) Survey method matters: online/ offline questionnaires and face-to-face or telephone interviews differ. Comput Hum Behav 71:172-180. https://doi.org/10.1016/j.chb.2017.02.006

Zolas N, Goldschlag N, Jarmin R et al. (2015) Wrapping it up in a person: examining employment and earnings outcomes for Ph.D. recipients. Science (80-) 350:1367-1371. https://doi.org/10.1126/science.aac5949

\section{Acknowledgements}

The authors wish to thank the Doctoral Researchers who participated in the present study, and the universities and individuals who helped to promote the U-DOC project. This research was jointly funded by Research England and the Office for Students (https://re.ukri.org/research/postgraduate-researchers/).

\section{Competing interests}

The authors declare no competing interests.

\section{Ethical approval}

This study was performed in line with the principles of the Declaration of Helsinki. Approval was granted by the University of Sussex Sciences and Technology CrossSchools Research Ethics Committee (C-REC) (Date: 19 December 2017; Reference: $\mathrm{ER} / \mathrm{CH} 283 / 9)$.

\section{Informed consent}

All individuals who took part in this study provided informed consent to participate and for their data to be used in research reports.

\section{Additional information}

Correspondence and requests for materials should be addressed to Clio Berry.

Reprints and permission information is available at http://www.nature.com/reprints

Publisher's note Springer Nature remains neutral with regard to jurisdictional claims in published maps and institutional affiliations.

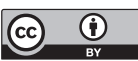

Open Access This article is licensed under a Creative Commons Attribution 4.0 International License, which permits use, sharing, adaptation, distribution and reproduction in any medium or format, as long as you give appropriate credit to the original author(s) and the source, provide a link to the Creative Commons license, and indicate if changes were made. The images or other third party material in this article are included in the article's Creative Commons license, unless indicated otherwise in a credit line to the material. If material is not included in the article's Creative Commons license and your intended use is not permitted by statutory regulation or exceeds the permitted use, you will need to obtain permission directly from the copyright holder. To view a copy of this license, visit http://creativecommons.org/ licenses/by/4.0/.

(c) The Author(s) 2021 